\title{
Evaluation of Transbronchial Lung Cryobiopsy Size and Freezing Time: A Prognostic Animal Study
}

\author{
Matthew Ing ${ }^{a}$ Rema A. Oliver ${ }^{b}$ Brian G.G. Oliver ${ }^{c}$ William R. Walsh ${ }^{b}$ \\ Jonathan P. Williamson ${ }^{d}$ \\ ${ }^{a}$ Faculty of Medicine, University of Notre Dame, ${ }^{b}$ Surgical and Orthopaedic Research Laboratories, University of \\ New South Wales Australia, Prince of Wales Clinical School, Prince of Wales Hospital, 'Woolcock Institute of Medical \\ Research and School of Life Sciences, University of Technology Sydney, and d Department of Respiratory Medicine, \\ Liverpool Hospital, Sydney, N.S.W., Australia
}

\section{Key Words}

Transbronchial cryobiopsy · Freezing time · Biopsy size ·

Animal · Sheep

\section{Abstract}

Background: Transbronchial lung biopsy using a cryoprobe is a novel way of sampling lung parenchyma. Correlation of freezing time with biopsy size and complications has not been evaluated in vivo. Objectives: The primary aim of the study is to evaluate the correlation between transbronchial cryobiopsy freezing time and size. The secondary aims are to evaluate histological quality of the biopsy and evaluate procedure-associated complications. Methods: Transbronchial lung cryobiopsies were obtained from two anaesthetised sheep using a 1.9-mm cryoprobe inserted into a flexible bronchoscope under fluoroscopic guidance. Freezing times ranged from 1 to $6 s(n=49)$. The cryobiopsies were evaluated histologically with respect to their size and quality. Complications of bleeding and pneumothorax were recorded. Results: The mean cross-sectional area of the cryobiopsy ranged from $4.7 \pm 2.1$ to $15.7 \pm 15.3 \mathrm{~mm}^{2}$. There was a significant positive correlation between increasing freezing time and cryobiopsy cross-sectional area $(p=0.028)$. All biopsies contained lung tissue with preserved parenchyma. Crush and freeze artefacts were not observed and tissue architecture was intact in all specimens. Small blood vessels and terminal bronchioles were observed in $88 \%$ of specimens. All cryobiopsies caused nil or mild haemorrhage with the exception of only 1 episode of severe haemorrhage at $6 \mathrm{~s}$ freezing time. Pneumothoraces occurred at 2, 5 and $6 \mathrm{~s}$ freezing time and required chest tube insertion. The most significant haemorrhage and pneumothoraces occurred at 5 and $6 \mathrm{~s}$. Our results suggest an initial freezing time of $3 \mathrm{~s} \mathrm{can}$ provide the maximal biopsy size while minimising major complications. Conclusion: The optimal transbronchial cryobiopsy freezing time is initially $3 \mathrm{~s}$. This time is associated with minimal complications and large artefact-free biopsies.

(c) 2016 S. Karger AG, Basel

\section{Introduction}

Bronchoscopic transbronchial lung biopsy is a commonly used technique to obtain tissue specimens from the peripheral lung to evaluate diffuse parenchymal infil-

\section{KARGER}

E-Mail karger@karger.com www.karger.com/res
(C) 2016 S. Karger AG, Basel

$0025-7931 / 16 / 0921-0034 \$ 39.50 / 0$
Dr. Matthew Ing

Suite 306, 2 Technology Place

Macquarie University Clinic

Sydney, NSW 2109 (Australia)

E-Mail 20110575@my.nd.edu.au 
trates and lung masses. Traditionally, transbronchial biopsies involve the use of flexible forceps to obtain specimens. However, there are several limitations of this technique including the small biopsy size, the biopsy only containing mucus or being very superficial, and the susceptibility to crush artefact. These hamper histopathological analysis and thereby limit diagnostic yield $[1,2]$.

Transbronchial cryobiopsy is a novel method that has the potential to increase the diagnostic yield of transbronchial lung biopsies. Through the cryoadhesive effect, large specimens that demonstrate unaltered and artefactfree morphology can be obtained [3-7]. Transbronchial cryobiopsy is a promising technique because both biopsy size and quality contribute to diagnostic value [8]. Early studies suggest that transbronchial cryobiopsy has a higher diagnostic yield with similar safety profiles to transbronchial forceps biopsies in interstitial lung disease [4, $6,7,9-13]$, lung transplantation rejection $[14,15]$ and transbronchial malignancy [16].

As the uptake of transbronchial cryobiopsies increases, the optimum technique is still being investigated. Uncertainty remains regarding the correct cryoprobe freezing time for optimum transbronchial cryobiopsies. Recent in vivo and ex vivo studies have provided conflicting reports of the correlation between biopsy weight and freezing time $[8,17]$. Cryoprobe freezing times for transbronchial cryobiopsies have ranged from 3 to $5 \mathrm{~s}[4,6,9,14,15]$, yet to date there has been no research into freezing times and its effect on in vivo transbronchial cryobiopsies. The primary aim of this animal study was to examine the relationship between the freezing time of transbronchial cryobiopsies and biopsy size. Secondary aims were to evaluate the risk of complications associated with each freezing time and the histological quality of cryobiopsy samples.

\section{Methods}

The study was conducted at the University of New South Wales Biological Resources Centre, at St George Hospital. The study was conducted between May 2014 and December 2014. The study was approved by the University of New South Wales Animal Care and Ethics Committee.

\section{Protocol}

The transbronchial lung cryobiopsies were obtained from two healthy, $90-\mathrm{kg}$ adult Border Leicester Merino cross sheep. The animals were anaesthetised using an intramuscular injection of tiletamine-zolazepam and maintained with isoflurane. A tracheotomy was performed and transbronchial cryobiopsies were obtained using a 2.8-mm channel flexible bronchoscope (BF-1TH180, EVIS EXERA II CLV-180, Olympus Corp., Tokyo, Japan) and a 1.9-mm flexible cryoprobe (Erbokryo CA, ERBE Tübingen, Germany).

Transbronchial Lung Cryobiopsy Size and Freezing Time
One trained operator (J.P.W.) performed the transbronchial cryobiopsies. The cryoprobe was advanced under fluoroscopic guidance towards the pleura until resistance was met where it was withdrawn approximately $10 \mathrm{~mm}$. The probe was then cooled using compressed carbon dioxide to achieve specific freezing times. The cryoprobe activation time was increased incrementally for 1 , $2,3,4,5$ or 6 s to achieve individual cryobiopsies. Between 6 and 11 cryobiopsies were performed for each freezing time. Following freezing, the bronchoscope and cryoprobe with attached biopsy were removed en bloc. The frozen specimen was thawed in normal saline and immediately fixed in $10 \%$ buffered formalin. The segmental bronchi were selected randomly in a distal to proximal fashion, while freezing times were selected in a non-randomised fashion due to the potential risk of haemorrhage with larger freezing durations.

Complication rates of haemorrhage and pneumothorax were recorded. Pneumothorax was assessed using fluoroscopic screening (BV300 C-Arm, Philips, The Netherlands) following each biopsy. Haemorrhage was graded based on the intervention required to achieve haemostasis. Grade 0: no haemorrhage, grade 1: suctioning, topical cold saline or adrenaline, grade 2: topical tranexamic acid, bronchial blocker, and grade 3: haemodynamic instability leading to cessation of the procedure. The sheep were not recovered from anaesthesia and were sacrificed with an overdose of pentobarbitone sodium to undergo autopsy and tissue harvest.

\section{Histology}

Biopsy samples were fixed in 10\% buffered formalin, embedded in paraffin and cut into $5-\mu \mathrm{m}$ sections for staining with haematoxylin and eosin. The samples were analysed and assessed by one blinded observer. Analysis and imaging of the cryobiopsies was performed under a light microscope (BX60 Microscope and DP71 Camera, Olympus Corp., Tokyo, Japan). Automated analysis of the total area of the biopsy specimens was measured with interactive circling of the biopsy using the computer program Image J (version 1.48, Maryland, USA) and expressed in $\mathrm{mm}^{2}$. Features of artefact (crush artefact, atelectasis and specimen fragmentation) were assessed manually, as well as the percentage of specimens containing terminal bronchioles, small arteries and alveolar tissue.

\section{Statistical Analysis}

All data were analyzed using statistical software (SPSS version 22 for Windows, IBM Corp., N.Y., USA). Data was analysed and expressed via descriptive statistics (means and standard deviations). A linear regression model was performed to determine the correlation between biopsy area and freezing time. A KruskalWallis with Dunn's post test was performed to compare cross-sectional areas of different freezing times. A p value $<0.05$ was considered statistically significant. Complications and histological specimen quality are described qualitatively.

\section{Results}

\section{Correlation between Freezing and Biopsy Size}

Forty-nine transbronchial cryobiopsies were taken with the $1.9-\mathrm{mm}$ cryoprobe. Nineteen and thirty cryobiopsies were taken in each sheep, respectively. The mean 
Table 1. Transbronchial cryobiopsy cross-sectional area

\begin{tabular}{llllllll}
\hline & \multicolumn{7}{c}{ Cryoprobe freezing time, s } \\
\cline { 2 - 7 } & 1 & 2 & 3 & 4 & 5 & 6 \\
\hline Number of biopsies & 8 & 8 & 11 & 9 & 7 & 6 \\
Cross-sectional area, $\mathrm{mm}^{2}$ & 5.5 & 4.7 & 6.5 & 7.1 & 9.0 & 15.7 \\
Standard deviation & 2.3 & 2.1 & 2.2 & 2.8 & 5.7 & 15.3 \\
\hline
\end{tabular}

Table 2. Complications associated with freezing times

\begin{tabular}{cllllll}
\hline Complications & \multicolumn{5}{l}{ Freezing time, s } \\
\cline { 2 - 7 } & 1 & 2 & 3 & 4 & 5 & 6 \\
\hline Haemorrhage & & & & & & \\
Grade 0 & $4(50)$ & $3(38)$ & $2(18)$ & $4(44)$ & $2(29)$ & $3(50)$ \\
Grade 1 & $4(50)$ & $5(62)$ & $9(82)$ & $5(56)$ & $5(71)$ & $2(33)$ \\
Grade 2 & 0 & 0 & 0 & 0 & 0 & 0 \\
Grade 3 & 0 & 0 & 0 & 0 & 0 & $1(17)$ \\
Pneumothorax & Nil & 1 & Nil & Nil & 1 & 1 \\
\hline
\end{tabular}

Figures in parentheses are percentages.

cross-sectional area of the cryobiopsies ranged from 4.7 \pm 2.1 to $15.7 \pm 15.3 \mathrm{~mm}^{2}$ (table 1 ). A strong correlation between freezing time and cryobiopsy area was demonstrated $(r=0.7387)$ (fig. 1$)$. There was a significant positive correlation between increasing freezing time and cross-sectional area of transbronchial cryobiopsies ( $\mathrm{p}=$ 0.028). This correlation was specifically demonstrated through the 5- and 6-second freezing times, as there was no statistically significant difference between cryobiopsy cross-sectional area at 1-4 s freezing times (fig. 2).

\section{Complications}

The majority of transbronchial cryobiopsies were associated with grade 0-1 haemorrhage (98\%) (table 2). There was only one grade 3 haemorrhage that occurred at $6 \mathrm{~s}$ causing sacrifice of the animal. Three pneumothoraces occurred at 2, 5 and $6 \mathrm{~s}$ freezing time and required chest drain insertion. The pneumothoraces at 5 and $6 \mathrm{~s}$ were larger on fluoroscopy and caused greater oxygen desaturation (50-60\%) than the pneumothorax occurring at $2 \mathrm{~s}$.

\section{Histology}

Transbronchial cryobiopsy preserved the lung parenchyma with all biopsies containing lung tissue. Crush artefact was not seen and there was minimal tissue frag-

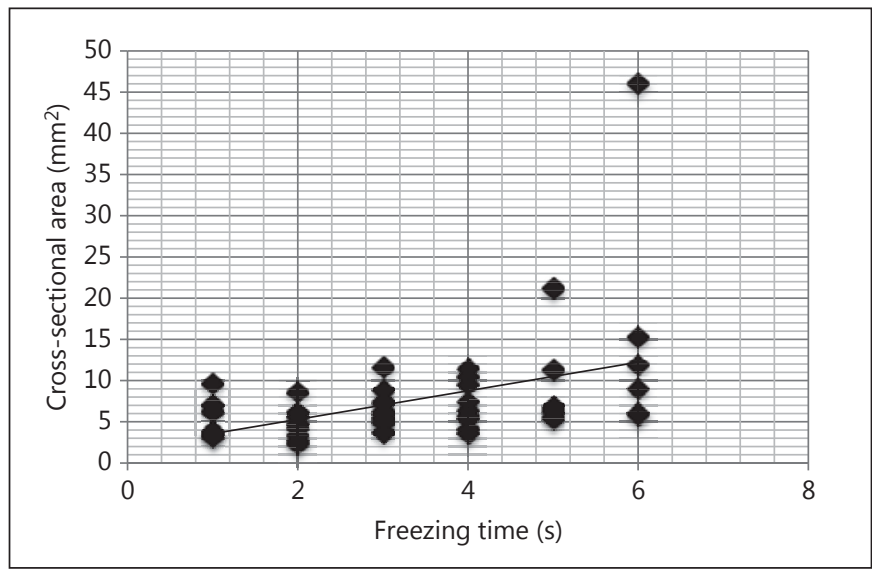

Fig. 1. Correlation between freezing time and transbronchial cryobiopsy cross-sectional area.

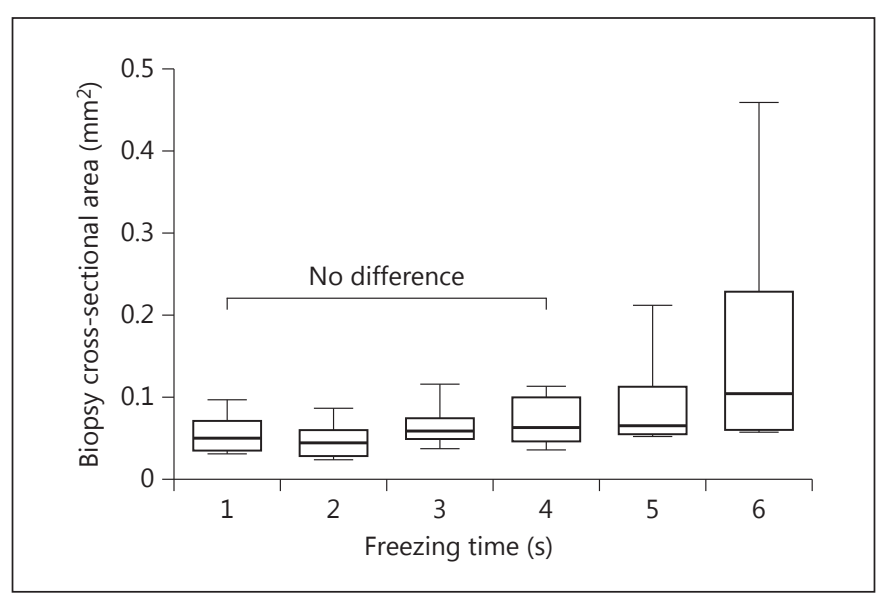

Fig. 2. Comparison of cross-sectional area of different freezing times.

mentation and atelectasis. Tissue architecture was intact in the majority of specimens (fig. 3). Small blood vessels and terminal bronchioles were observed in $88 \%$ of all specimens, while alveolar tissue was identified in $92 \%$ of all specimens (table 3).

\section{Discussion}

This is the first study to examine the relationship between in vivo transbronchial cryobiopsy size and freezing time. We were able to demonstrate in sheep a significant positive correlation between 1.9-mm cryoprobe freezing times and transbronchial cryobiopsy cross-sectional area. 
Table 3. Histological quality of cryobiopsy specimen

\begin{tabular}{lllllll}
\hline Histology & \multicolumn{7}{l}{ Freezing time, s } \\
\cline { 2 - 7 } & 1 & 2 & 3 & 4 & 5 & 6 \\
\hline Number of biopsies & 8 & 8 & 11 & 9 & 7 & 6 \\
Small arterioles & $7(88)$ & $7(88)$ & $9(82)$ & $8(89)$ & $6(86)$ & $6(100)$ \\
Terminal bronchioles & $7(88)$ & $7(88)$ & $9(82)$ & $8(89)$ & $6(86)$ & $6(100)$ \\
Alveolar tissue & $7(88)$ & $7(88)$ & $10(91)$ & $8(89)$ & $7(100)$ & $6(100)$ \\
\hline
\end{tabular}

Figures in parentheses are percentages.

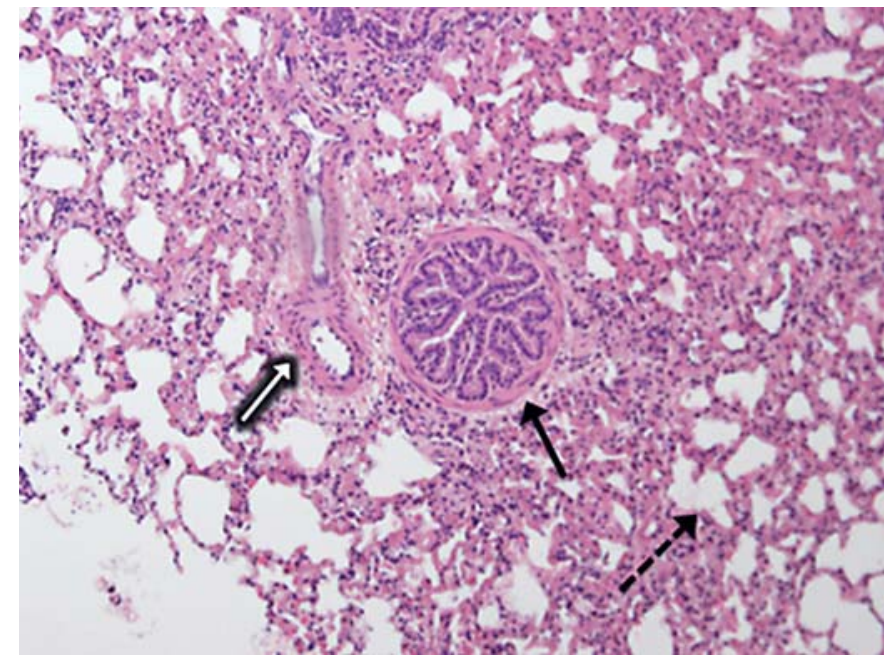

Fig. 3. Preserved tissue architecture of transbronchial lung cryobiopsy. Five-micrometer section stained in haematoxylin and eosin. Preserved tissue architecture without crush artefact. Normal alveoli with minimal atelectasis (dotted arrow). Terminal bronchiole (black arrow) and arteriole (white arrow) are preserved.

There was no statistical difference in cryobiopsy crosssectional area between 1-4 s freezing time and serious complications occurred only at 5 and $6 \mathrm{~s}$. Based on practicality and safety, we recommend an initial freezing time of $3 \mathrm{~s}$. Technically, a 3 -second freezing time period is easily achievable, provides a large biopsy size whilst creating a safety threshold from complications. This has implications in guiding the optimal application of transbronchial cryobiopsies in human populations.

Due to the novel nature of this study, comparison of freezing time and cryobiopsy area to previous literature is limited. In one in vitro study, non-perfused porcine lung demonstrated a strong positive correlation between biopsy weight, diameter of the probe and freezing time [17]. However, for endobronchial samples, one in vivo pro-

Transbronchial Lung Cryobiopsy Size and Freezing Time spective animal study found only a moderate correlation between biopsy weight and specimen diameter and the freezing period [8]. This inconsistency may be explained by the different nature of structures located in central airways for endobronchial applications of the cryoprobe, when compared to peripheral lung parenchyma, which we sampled. It has been shown that cryosensitivity depends on microcirculation and water content of the tissue [17]. Areas with higher rates of collagen, cartilage or poorly vascularised tissue (such as the proximal bronchial wall) are less susceptible to the cryoadhesive effect [18].

In terms of biopsy size, a similar transbronchial cryobiopsy cross-sectional area has been achieved for freezing times of 3-4 $\mathrm{s}$ with a $2.4-\mathrm{mm}$ cryoprobe $\left(10-15.11 \mathrm{~mm}^{2}\right)$ and $5 \mathrm{~s}$ freezing $\left(17.1 \pm 10.7 \mathrm{~mm}^{2}\right)[4,6,9,14]$. The significant positive correlation between freezing time and cross-sectional cryobiopsy area can be explained by the concentric expansion of the frozen zone that radiates from the tip of the cryoprobe [2]. Theoretically, by increasing freezing times, a larger surface area and subsequently larger biopsies can be generated.

This study demonstrated the variable cross-sectional area and a high degree of standard deviation with freezing times. This variability in cross-sectional area has also been evident in previous studies $[6,9]$. Despite the use of fluoroscopic guidance, flexible bronchoscopy and standardised placement of the cryoprobe, the operator cannot consistently ensure the placement of the probe is perpendicular (optimal) to the mucosa of the segmental bronchus. There is natural variability in placement and lateral application of the cryoprobe was observed in multiple cases [8]. As the cryobiopsy area is highly dependent on the contact area between the cryoprobe and the tissue $[17,18]$, this may explain the considerable variability seen in cross-sectional area especially observed with increasing freezing time.

Our study noted a high histological quality of transbronchial cryobiopsies. Cryobiopsies have demonstrated significantly larger size and alveolar sampling, lower evi- 
dence of artefact, preservation of tissue architecture and greater ability for immunohistochemical processing in comparison to transbronchial forceps biopsy $[4,6,10-12$, $14,15]$. These unique characteristics of transbronchial cryobiopsy have resulted in higher diagnostic yield in parenchymal pulmonary lesions [16], identification of lung transplant rejection $[14,15]$ and support the utility of transbronchial biopsies in interstitial lung diseases, an area where bronchoscopic biopsies have traditionally been considered to have a low yield [4, 6, 10-12]. Freezing times that provide the largest artefact-free specimen will therefore likely maximise diagnostic yield.

There have been very few studies evaluating the safety of transbronchial cryobiopsies when compared to forceps biopsy. One study found no difference in the safety profiles between transbronchial forceps and cryobiopsy in regards to pneumothorax, bleeding or the need for hospitalization in diffuse lung disease and lung transplant patients [13]. Haemorrhage and pneumothorax represent the most common complications and we found these complications were associated with freezing times of 5 and $6 \mathrm{~s}$. Despite the increasing size of the cryobiopsies with freezing time, only one grade 3 haemorrhage occurred during sampling. Previous literature has suggested that procedural sedation and intubation may aid in the management and control of severe haemorrhage [19]. The low rate of significant haemorrhage may be due to the appearance of intra- and extracellular ice crystals causing temporary vasoconstriction [18]. In the 49 biopsies performed, only 3 significant pneumothoraces occurred, all resolving with intercostal catheter insertion. However, there was difficulty drawing a definitive conclusion, as subsequent pneumothorax could not be assessed after intercostal catheter insertion. Nevertheless, both complications have been reported to occur at similar rates for both transbronchial cryobiopsy and forceps biopsy $[4,6,14,15]$.

The use of a large animal model and standardized operating protocol adds to the strength and reproducibility of the study. Our sheep model has direct applicability to human models. Sheep have airway structures and functions that closely resemble human lungs $[20,21]$. Due to these similarities, sheep have been used to model human lung pathology such as emphysema, asthma and chronic bronchitis [20]. Therefore, extrapolation of results that support $3 \mathrm{~s}$ as the optimal initial freezing time in sheep is likely to be valid for human transbronchial cryobiopsies. It may guide future research protocols in human studies and its end use as a diagnostic tool in patients with different lung pathology.

However, there remain particular limitations associated with our study. Despite the strength of airway and parenchymal similarities, care must be exercised in drawing conclusions from sheep models. Furthermore, a larger sample size over multiple sites may help further delineate differences between cross-sectional area and particular freezing times. This larger sample size may also potentially help explore the relationship between longer freezing times and the higher risk of complications.

This research adds to the growing body of evidence regarding the safe application of transbronchial cryobiopsies. We recommend the initial use of $3 \mathrm{~s}$ freezing time and subsequent use of $4 \mathrm{~s}$ freezing time if adequate samples are not initially obtained. This timeframe maintains optimal biopsy size while minimising common complications.

\section{Acknowledgements}

The cryoprobe was donated by ERBE Germany. Olympus Australia provided the animal-dedicated bronchoscopy equipment. Intercostal catheters were donated by Cook Medical.

\section{Disclosure Statement}

There are no conflicts of interest to declare.

\section{References}

Schumann C, Hetzel J, Babiak AJ, Merk T, Wibmer T, Moller P, Lepper PM, Hetzel M: Cryoprobe biopsy increases the diagnostic yield in endobronchial tumor lesions. J Thorac Cardiovasc Surg 2010;140:417-421.

-2 Hetzel J, Eberhardt R, Herth FJ, Petermann C, Reichle G, Freitag L, Dobbertin I, Franke KJ, Stanzel F, Beyer T, Moller P, Fritz P, Ott G, Schnabel PA, Kastendieck H, Lang W, Morresi-Hauf AT, Szyrach MN, Muche R, Shah PL, Babiak A, Hetzel M: Cryobiopsy increases the diagnostic yield of endobronchial biopsy: a multicentre trial. Eur Respir J 2012;39:685690.

- 3 Hetzel J, Hetzel M, Hasel C, Moeller P, Babiak A: Old meets modern: the use of traditional cryoprobes in the age of molecular biology. Respiration 2008;76:193-197.

4 Babiak A, Hetzel J, Krishna G, Fritz P, Moeller P, Balli T, Hetzel M: Transbronchial cryobiopsy: a new tool for lung biopsies. Respiration 2009;78:203-208. 
5 Aktas Z, Gunay E, Hoca NT, Yilmaz A, Demirag F, Gunay S, Sipit T, Kurt EB: Endobronchial cryobiopsy or forceps biopsy for lung cancer diagnosis. Ann Thorac Med 2010; 5:242-246.

6 Pajares V, Puzo C, Castillo D, Lerma E, Montero MA, Ramos-Barbón D, Amor-Carro O, Gil de Bernabé A, Franquet T, Plaza V, Hetzel J, Sanchis J, Torrego A: Diagnostic yield of transbronchial cryobiopsy in interstitial lung disease: a randomized trial. Respirology 2014; 19:900-906.

$>7$ Torrego A, Pajares V: Transbronchial cryobiopsy in diffuse lung disease. Curr Respir Care Rep 2014;3:7-12.

-8 Franke KJ, Theegarten D, Hann von Weyhern C, Nilius G, Brueckner C, Hetzel J, Hetzel M, Ruhle KH, Enderle MD, Szyrach MN: Prospective controlled animal study on biopsy sampling with new flexible cryoprobes versus forceps: evaluation of biopsy size, histological quality and bleeding risk. Respiration 2010; 80:127-132.

-9 Griff S, Ammenwerth W, Schonfeld N, Bauer TT, Mairinger T, Blum TG, Kollmeier J, Gruning W: Morphometrical analysis of transbronchial cryobiopsies. Diagn Pathol 2011;6:53.
10 Casoni GL, Tomassetti S, Cavazza A, Colby TV, Dubini A, Ryu JH, Carretta E, Tantalocco P, Piciucchi S, Ravaglia C, Gurioli C, Romagnoli M, Gurioli C, Chilosi M, Poletti V: Transbronchial lung cryobiopsy in the diagnosis of fibrotic interstitial lung diseases. PLoS One 2014;9:e86716.

11 Kropski J, Pritchett J, Mason W, Sivarajan L, Gleaves L, Johnson J, Lancaster L, Lawson W, Blackwell T, Steele M, Loyd J, Rickman O: Bronchoscopic cryobiopsy for the diagnosis of diffuse parenchymal lung disease. PLoS One 2013;8:e78674.

12 Fruchter O, Fridel L, El Raouf BA, AbdelRahman N, Rosengarten D, Kramer MR: Histological diagnosis of interstitial lung diseases by cryo-transbronchial biopsy. Respirology 2014;19:683-688

13 Gershman E, Fruchter O, Benjamin F, Nader AR, Rosengarten D, Rusanov V, Fridel L, Kramer MR: Safety of cryo-transbronchial biopsy in diffuse lung diseases: analysis of three hundred cases. Respiration 2015;90:40-46.

14 Fruchter O, Fridel L, Rosengarten D, Raviv Y, Rosanov V, Kramer MR: Transbronchial cryo-biopsy in lung transplantation patients: first report. Respirology 2013;18:669-673.

-15 Yarmus LDOF, Akulian JMD, Gilbert CDO, Illei PMD, Shah PMD, Merlo CMD, Orens JMDF, Feller-Kopman DMDF: Cryoprobe transbronchial lung biopsy in patients after lung transplantation: a pilot safety study. Chest 2013;143:621-626.
16 Schuhmann M, Bostanci K, Bugalho A, Warth A, Schnabel PA, Herth FJ, Eberhardt R: Endobronchial ultrasound-guided cryobiopsies in peripheral pulmonary lesions: a feasibility study. Eur Respir J 2014;43:233-239.

17 Franke KJ, Szyrach M, Nilius G, Hetzel J, Hetzel M, Ruehle KH, Enderle MD: Experimental study on biopsy sampling using new flexible cryoprobes: influence of activation time, probe size, tissue consistency, and contact pressure of the probe on the size of the biopsy specimen. Lung 2009;187:253-259.

18 Vergnon JM, Huber RM, Moghissi K: Place of cryotherapy, brachytherapy and photodynamic therapy in therapeutic bronchoscopy of lung cancers. Eur Respir J 2006;28:200218.

19 Poletti V, Hetzel J: Transbronchial cryobiopsy in diffuse parenchymal lung disease: need for procedural standardization. Respiration 2015;90:275-278.

20 Meeusen EN, Snibson KJ, Hirst SJ, Bischof RJ: Sheep as a model species for the study and treatment of human asthma and other respiratory diseases. Drug Discov Today Dis Models 2009;6:101-106.

21 Scheerlinck J-P Y, Snibson KJ, Bowles VM, Sutton P: Biomedical applications of sheep models: from asthma to vaccines. Trends Biotechnol 2008;26:259-266. 